\title{
Gravitational radiation from young magnetars: Preliminary results
}

\author{
C. Palomba ${ }^{1,2}$ \\ 1 Dipartimento di Fisica "G. Marconi", Università di Roma "La Sapienza", Piazzale A. Moro 5, 00185 Roma, \\ Italy \\ 2 Sezione INFN Roma1, Piazzale A. Moro 5, 00185 Roma, Italy
}

Received 6 September 2000 / Accepted 6 November 2000

\begin{abstract}
We consider newborn magnetars as sources of gravitational radiation. We focus attention on the early stages of their life, when, due to the distortion induced by the strong magnetic field, a large amount of radiation could be emitted. Many aspects of the characteristics and evolution of newborn neutron stars endowed with a large magnetic field are still poorly known, and we perform calculations making some simplifying assumptions. Our preliminary results suggest that this kind of source deserves deeper study.
\end{abstract}

Key words. magnetic fields - gravitational waves - stars: neutron

\section{Introduction}

There is increasing evidence that soft gamma-ray repeaters $(S G R)$ and anomalous X-ray pulsars $(A X P)$ are magnetars (Thompson \& Duncan 1996). They are defined as neutron stars whose dipole magnetic field is greater than the critical value $B_{\mathrm{c}}=\frac{m_{\mathrm{e}}^{2} c^{3}}{e h}=4.410^{13} \mathrm{G}$, above which the electrons Landau energy exceeds their rest energy. In such a regime, many new effects take place in the star magnetosphere, such as magnetic lensing, photon splitting and merging and suppression of photon-electron scattering; see Duncan (1999) for more details. In magnetars, magnetic energy, rather than rotational energy as in normal pulsars, is the main source of free energy. According to the current theory (Duncan \& Thompson 1992), a large magnetic field, of the order of $10^{14} \sim 10^{16} \mathrm{G}$ could be produced soon after the proto-neutron star formation, due to a $\alpha-\Omega$ dynamo mechanism, which could be at work if the initial period is in the millisecond range. The strong magnetic field can induce large deformations in the star, which could be, then, a copious source of gravitational waves.

In this paper we consider the dynamic evolution of newborn magnetars and estimate the detectability by interferometric detectors of the gravitational waves emitted, under various hypotheses of the strength of the magnetic field and its structure inside the star. Note that the potential interest of young magnetars as sources of gravitational radiation has been suggested also by Konno et al. (2000).

Send offprint requests to: C. Palomba,

e-mail: cristiano.palomba@roma1.infn.it
Many aspects of the problem are still unknown and we make some simplifying, but reasonable, assumptions. Our calculations must be considered as a starting point for a more detailed analysis.

The plan of the paper is as follows. In Sect. 2 we briefly describe the theory of magnetars and the characteristics of observed $A X P$ and $S G R$. In Sect. 3 we give the basic relations describing the deformation of a fluid star under the effect of a magnetic field. In Sect. 4 we determine the dynamic evolution of a newborn magnetar under the effect of both magnetic and gravitational torques. In Sect. 5 we calculate the emission of gravitational waves and estimate their detectability by interferometric detectors. Finally, in Sect. 6 conclusions and open questions are discussed.

\section{The model for magnetars}

Immediately after the collapse of a massive star $(M>$ $\left.9 M_{\odot}\right)$, a proto-neutron star forms. It is rather extended (radius $R \sim 100 \mathrm{~km})$, hot $\left(T \sim 10^{12} \mathrm{~K}\right)$ and differentially rotating. It cools and contracts on a timescale of $\sim 20 \mathrm{~s}$, due to neutrino diffusion. Turbulent convection in the interior of the star takes place in this initial phase but helical convection and differential rotation can produce an amplification of the magnetic field, through an $\alpha-\Omega$ dynamo, if the initial period is small enough. According to Duncan \& Thompson (1992) (see also Duncan \& Thompson 1995), an initial period $P<3 \mathrm{~ms}$ is required in order to produce an inner magnetic field as large as $10^{16} \div 10^{17} \mathrm{G}$. The amplification of the magnetic field is at the expense of the differential rotation so that after deleptonization we have 
a hot and strongly magnetized neutron star, with radius $R \sim 10 \mathrm{~km}$, temperature $T \sim 10^{11} \mathrm{~K}$ and nearly uniform rotation. Goussard et al. (1998) have shown that, starting from a differentially rotating proto-neutron star, the initial period of the neutron star, $P_{0}$, cannot be smaller than $\simeq 1.7 \mathrm{~ms}$. We will assume $P_{0}=2 \mathrm{~ms}$. The subsequent evolution is dominated by modified URCA processes, which cause a decrease of the temperature to $T \sim 10^{10} \mathrm{~K}$ in about $1 \mathrm{~min}$. At this point a solid crust begins to form. After one year, when the temperature reaches $\sim 10^{9} \mathrm{~K}$, the transition of the star interior to a superfluid takes place.

6 objects have been classified as anomalous X-ray pulsars $(A X P)$ and 3 as soft gamma-ray repeaters $(S G R)$, and several candidates have been identified (see Table 1). Both categories are characterized by spin period in the range $5-12 \mathrm{~s}$ and high spin-down rates $\left(\dot{P} \sim 510^{-13} \div\right.$ $\left.10^{-10} \mathrm{~s} / \mathrm{s}\right)$. These stars show a quiescent X-ray luminosity in the range $10^{34} \div 10^{36} \mathrm{erg} / \mathrm{s}$ and soft X-ray spectra $\left(T_{\mathrm{c}}<1 \mathrm{keV}\right)$. Some of them are associated with young supernova remnants. Moreover, the $S G R$ group show recurrent energetic short gamma bursts with a timescale of months to years. It has been suggested that $A X P$ stars could be $S G R s$ in a phase of seismic inactivity (Thompson et al. 1999). Assuming that their spin-down is due to dipolar magnetic braking, the strength of the magnetic field is given by

$$
B=3.210^{19} \sqrt{P \dot{P}}
$$

Using typical values of $P, \dot{P}$ measured from observed $A X P s$ and $S G R s$, we obtain strengths of the magnetic field in the range $10^{14} \div 10^{15} \mathrm{G}^{1}$, see Table 1 . Soon after their formation, the exterior dipole energy exceeds the rotational energy and becomes the dominant free source of energy in magnetars. The rotational energy cannot explain the persistent X-ray emission of such objects and it has been argued that they could be powered by the decay of the magnetic field. This can happen in several ways (Goldreich \& Reisenegger 1992): through ambipolar diffusion, in the irrotational or solenoidal modes, or through the Hall cascade. During these diffusive processes, sudden releases of magnetic energy probably occur, due for instance to crustal fractures, and could be the origin of the bursts observed in $S G R$. According to Colpi et al. (2000), the period clustering shown by $A X P$ and $S G R$, and the association, observed in some cases, with relatively young supernova remnants, support a time-scale for magnetic field decay of $\sim 10^{4} \mathrm{yr}$, which can be satisfied by the fast Hall cascade occurring in magnetars whose magnetic field is confined in the crust. If this is the dominant channel for field decay, then the initial strength of the magnetic field would be much larger than we infer today and initial values of $B \sim 10^{15}-10^{16} \mathrm{G}$ can be assumed.

\footnotetext{
${ }^{1}$ Note, however, that for $S G R$, wind-aided spin-down probably contributes to the measured $\dot{P}$ and therefore the corresponding $B$ must be considered as an upper limit.
}

\section{Deformation induced by a magnetic field}

In this section, we deal with the distortion of the star induced by a magnetic field, relying mainly on the work of Bonazzola \& Gourgoulhon (1996) and Bocquet et al. (1995). Let us consider a fluid neutron star endowed with a magnetic field, with the magnetic axis in an arbitrary direction. The star deforms under the Lorentz force exerted by the field and the amount of deformation depends on the strength of the field, on its structure in the interior of the star, and on the neutron star equation of state. The resulting ellipticity, which in the Newtonian limit is defined as $\epsilon=\left(I_{z}-I_{x}\right) / I_{z}$, where $I_{z}, I_{x}$ are the momenta of inertia with respect to the rotation axis and along a direction perpendicular to it, is proportional to the square of the magnetic dipole moment. It can be expressed, in Gauss units, as

$\epsilon=\beta\left(\frac{R^{8}}{4 G I_{z}^{2}}\right) B^{2}$,

where $R$ is the star radius and $\beta$ is the distortion parameter, which depends on the star equation of state and on the geometry of the field inside the star. For example, for an incompressible fluid with a uniform internal magnetic field, $\beta=1 / 5$ (Bonazzola \& Gourgoulhon 1996), but $\beta$ can have larger values for other configurations, as we will discuss in the following of the section. Equation (2) can be re-written as

$$
\begin{aligned}
\epsilon= & 3.7510^{-7} \beta\left(\frac{R}{10 \mathrm{~km}}\right)^{8} \\
& \times\left(\frac{I_{z}}{10^{38} \mathrm{~kg} \mathrm{~m}^{2}}\right)^{-2}\left(\frac{B}{10^{14} \mathrm{G}}\right)^{2} .
\end{aligned}
$$

The maximum strength of the magnetic field resulting in a stable configuration corresponds to the situation in which the magnetic pressure is equal to the fluid pressure (e.g. Bocquet et al. 1995) and is in the range $B_{\max } \simeq 410^{17} \sim$ $10^{18} \mathrm{G}$, depending on the equation of state used. The presence of a very strong magnetic field seems to increase the stability of the star configuration ${ }^{2}$ but a complete study of dynamical and secular instabilities in strongly deformed stars has yet to be performed. Bonazzola \& Gourgoulhon (1996) have considered several magnetic configurations in the interior of a neutron star, described by a realistic equation of state, calculating the corresponding value of the distortion parameter $\beta$. In the case of a perfectly conducting interior, they find $\beta$ varies between 1 , for a uniform field, to $\sim 9$ for a field confined in the external layers of the star. Greater values of $\beta$, of the order of hundreds, are obtained if the stellar interior is a superconductor of type $\mathrm{I}^{3}$. Still greater values, of the order of thousands, have

\footnotetext{
${ }^{2}$ For instance, Bocquet et al. find an increase in the massshed limit for the case of magnetic axis parallel to the rotation axis.

${ }^{3}$ Note that the transition to superfluid occurs when the temperature drops below $\sim 10^{9} \mathrm{~K}$, i.e. after the formation of the solid crust.
} 
Table 1. List of identified soft gamma-ray repeaters $(S G R)$ and anomalous X-ray pulsars $(A X P)$. For each object, the period $P$, the period derivative $\dot{P}$ and the corresponding magnetic field $B$, as derived from Eq. (1), are given. In the last column, the association with a supernova remnant is also reported. Adapted from Mereghetti (1999)

\begin{tabular}{|c|c|c|c|c|}
\hline AXP & $P[\mathrm{~s}]$ & $\dot{P}[\mathrm{~s} / \mathrm{s}]$ & $B[\mathrm{G}]$ & $S N R$ \\
\hline $1 \mathrm{E} 1048.1-5937$ & 6.45 & {$[1.5-4] 10^{-11}$} & {$[3.1-5.1] 10^{14}$} & \\
\hline $1 \mathrm{E} 2259+586$ & 6.98 & $510^{-13}$ & $610^{13}$ & G109.1-0.1 \\
\hline $4 \mathrm{U} 0142+61$ & 8.69 & $210^{-12}$ & $1.310^{14}$ & \\
\hline RXS J170849-4009 & 11.0 & $210^{-11}$ & $4.710^{14}$ & \\
\hline $1 \mathrm{E} 1841-045$ & 11.77 & $4.110^{-11}$ & $710^{14}$ & Kes 73 \\
\hline AX J1845.0-0300 & 6.97 & & & G29.6+0.1 \\
\hline SGR & & & & N49 (LMC) \\
\hline SGR 0526-66 & 8.1 & & $810^{14}$ & G10.0 - 0.3 \\
\hline SGR 1806-20 & 7.48 & $8.310^{-11}$ & {$[5.1-8.6] 10^{14}$} & G42.8+0.6 \\
\hline SGR 1900+14 & 5.16 & {$[5-14] 10^{-11}$} & &
\end{tabular}

been found for a stochastic magnetic field, which they have simulated with counter-rotating electric currents. The occurrence of such high values of $\beta$ means that the strength of the magnetic field somewhere inside the star is much larger than the dipole field outside. This could be the case in newborn magnetars, which are characterized by turbulent convection immediately after their birth. Moreover, as temperature decreases, the star equation of state softens and this, according to the results of Konno et al. (2000) for politropic equations of state, should furtherly increase $\beta$. Once the crust is solidified, if electric currents are mainly located in the outer layer, larger values of $\beta$, with respect to the uniform case, are produced. In conclusion, we can reasonably expect that distortion parameters $\beta>10 \sim 100$ can be produced in the early phases of the evolution of magnetars.

It may be interesting to compare the star deformation induced by a magnetic field with that due to rotation in a fluid star. Deformation due to rotation can be expressed through the corresponding ellipticity (Chandrasekhar 1969)

$\epsilon_{\nu}=2 \pi^{2} \gamma\left(\frac{R^{5}}{G I_{z}}\right) \nu^{2}$

where $\nu$ is the star rotation frequency and $\gamma$ is a coefficient which Konno et al. (2000) showed to be $\sim 1$ for politropic equations of state and for a wide range of values for the relativistic factor $\frac{M}{R}, M$ being the gravitational mass of the star and $R$ its circumferential radius. The ratio between Eqs. $(2,4)$ is

$$
\begin{aligned}
r= & \frac{\epsilon}{\epsilon_{\nu}}=1.310^{-4} \frac{\beta}{\gamma}\left(\frac{R}{10 \mathrm{~km}}\right)^{3}\left(\frac{I_{z}}{10^{38} \mathrm{~kg} \mathrm{~m}^{2}}\right)^{-1} \\
& \times\left(\frac{B}{10^{14} \mathrm{G}}\right)^{2}\left(\frac{\nu}{100 \mathrm{~Hz}}\right)^{-2} .
\end{aligned}
$$

In Fig. 1 we plotted the ratio $\epsilon / \epsilon_{\nu}$ as a function of $\nu$ for different values of $\log \left(\beta \cdot B^{2}\right)$. We see that for magnetic fields of the order of $10^{15}-10^{16} \mathrm{G}$, even for rather fast

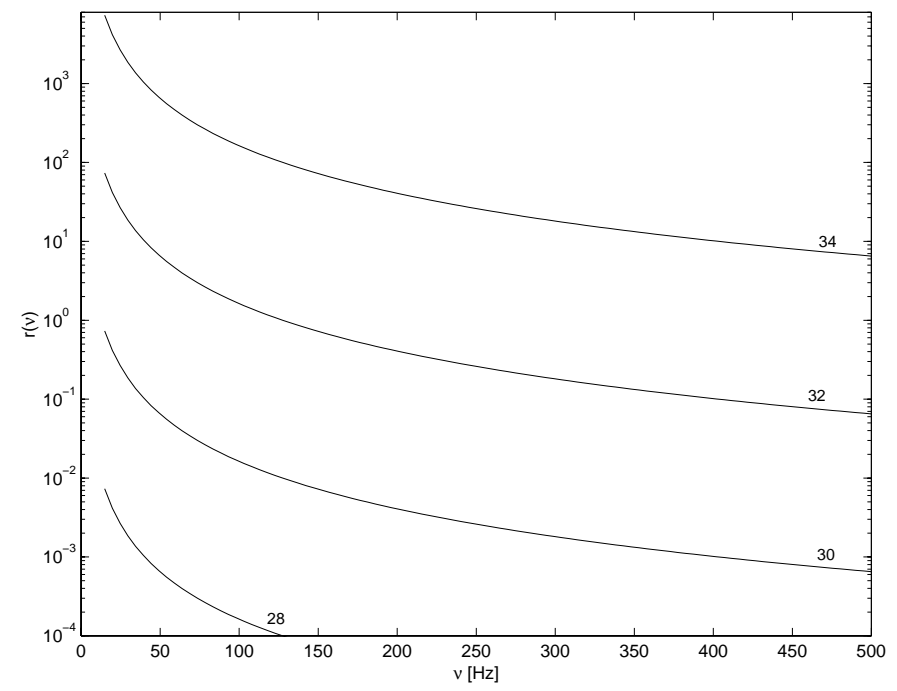

Fig. 1. Ratio $r=\frac{\epsilon}{\epsilon_{\nu}}$ between the ellipticity induced by the magnetic field and that due to rotation, as a function of the rotation frequency. Different curves correspond to different values of $\log \left(\beta \cdot B^{2}\right)$

rotation and small values of $\beta$, the magnetic distortion is dominant with respect to that induced by rotation. In this case, the magnetic axis of the star coincides with one of its principal axes of inertia.

\section{Dynamic evolution of newborn magnetars}

In this section, we describe the dynamic evolution of a newborn fluid magnetar, with an initial angular frequency $\nu_{0}=500 \mathrm{~Hz}$, which spins down due to the action of the electromagnetic and gravitational torques.

The law describing gravitational spin-down can be easily found from the expression of the power emitted in gravitational waves:

$\dot{E}=-\frac{G}{5 c^{5}}\left\langle\frac{\mathrm{d}^{3} \mathcal{I}_{i j}}{\mathrm{~d} t^{3}} \frac{\mathrm{d}^{3} \mathcal{I}_{i j}}{\mathrm{~d} t^{3}}\right\rangle=I \Omega \dot{\Omega}$, 


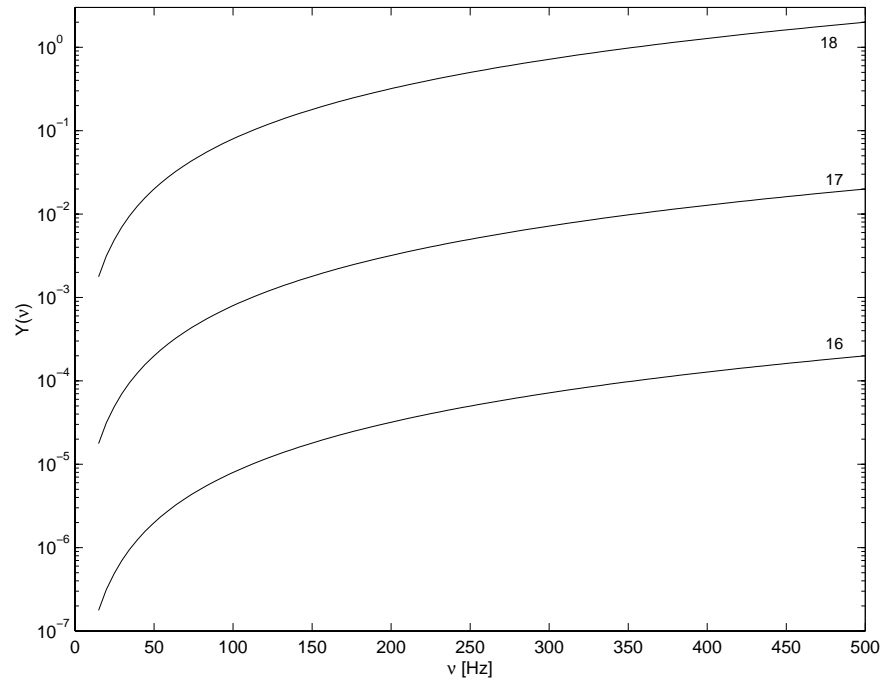

Fig. 2. Ratio $Y=\frac{\dot{\Omega}_{\mathrm{gw}}}{\Omega_{\mathrm{em}}}$ as a function of the rotation frequency, $\nu$. Different curves correspond to different values of $\log (\beta \cdot B)$

where $\mathcal{I}_{i j}$ is the mass quadrupole moment tensor and an average over time has been performed. Bonazzola \& Gourgoulhon (1996) found that

$\mathcal{I}_{i j}=\frac{1}{3} I_{z} \epsilon$
$\times\left(\begin{array}{ccc}3 \sin ^{2} \alpha \sin ^{2} \Omega t-1 & -3 / 2 \sin ^{2} \alpha \sin 2 \Omega t & -3 / 2 \sin 2 \alpha \sin \Omega t \\ -3 / 2 \sin ^{2} \alpha \sin 2 \Omega t & 3 \sin ^{2} \alpha \cos ^{2} \Omega t-1 & 3 / 2 \sin 2 \alpha \cos \Omega t \\ -3 / 2 \sin 2 \alpha \sin \Omega t & 3 / 2 \cos \alpha \cos \Omega t & 3 \cos ^{2} \alpha-1\end{array}\right)$,

where $\alpha$ is the angle between the rotation and the magnetic axes. From the last relation and Eq. (6) we obtain

$\dot{\Omega}_{\mathrm{gw}}=-2 \frac{G}{c^{5}} I_{z} \epsilon^{2} \sin ^{2} \alpha\left(\frac{1}{5}+3 \sin ^{2} \alpha\right) \Omega^{5}$.

The electromagnetic contribution to the spin-down can be expressed, for a dipolar field, as

$\dot{\Omega}_{\mathrm{em}}=-\frac{2}{3 c^{3}} \frac{R^{6}}{I_{z}} B^{2} \sin ^{2} \alpha \Omega^{3}$.

Using Eq. (2), the ratio can be written as

$$
\begin{aligned}
Y= & \frac{\dot{\Omega}_{\mathrm{gw}}}{\dot{\Omega}_{\mathrm{em}}}=4.910^{-10} \beta^{2}\left(\frac{R}{10 \mathrm{~km}}\right)^{10}\left(\frac{I_{z}}{10^{38} \mathrm{~kg} \mathrm{~m}^{2}}\right)^{-2} \\
& \times\left(\frac{B}{10^{14} \mathrm{G}}\right)^{2}\left(\frac{1}{5}+3 \sin ^{2} \alpha\right)\left(\frac{\nu}{200 \mathrm{~Hz}}\right)^{2} .
\end{aligned}
$$

It occurs that for large values of the product $\beta \cdot B$, the emission of gravitational waves can be the main mechanism for spin-down for a few seconds during the early phases of evolution. In most cases, however, the spin-down is dominated by $\dot{\Omega}_{\mathrm{em}}$. The function $Y(\nu)$ is plotted in Fig. 2. We can also calculate the corresponding braking index which is given by Palomba (2000) as

$n=\frac{3+5 Y}{1+Y}$.
The braking index can be initially close to 5 if spin-down is dominated by the emission of gravitational waves, but then it rapidly decreases toward $n=3$.

Integrating the equation $\dot{\Omega}=\dot{\Omega}_{\mathrm{gw}}+\dot{\Omega}_{\mathrm{em}}$ we can easily calculate the characteristic spin-down time for fixed values of $B$ and $\beta$. We find that the spin-down time (to a final frequency $\nu_{\mathrm{f}}=10 \mathrm{~Hz}$ ) is $t_{\mathrm{sd}} \sim 1.3 \mathrm{yr}, 5 \mathrm{~d}, 1 \mathrm{~h}$ respectively for $B=10^{14}, 10^{15}, 10^{16} \mathrm{G}$.

On the other hand, $\sim 1$ min after the birth of the magnetar the solid crust begins to form. In estimating the detectability of gravitational radiation, we will treat these two phases separately. In fact, the formation of the crust is a transient process which may perturb the smooth gravitational signal emitted during the initial, "fluid", phase. If we cannot accurately model this transient, by integrating over long time periods we worsen the signal-to-noise ratio. Once the crust is formed, the evolution of the star is given by the same equations as before. What changes is that, for temperatures greater than about one tenth of the melting point, i.e. $\sim 10^{9} \mathrm{~K}$, the crust, due to the stresses induced by the large magnetic field, undergoes a plastic deformation rather than sudden fractures, with a gradual increase of the ellipticity, $\epsilon^{4}$. For colder stars, the crust starts breaking under the effect of magnetic stresses and this probably produces glitches (Ruderman 1991, 1991). However, we are not interested in this phase of gravitational emission, because it starts about 1 yr after the birth of the magnetar, when the frequency of the signals emitted is outside the sensitivity band of interferometers. As we will see in the next section, for increasing magnetic fields, more and more of the gravitational radiation is emitted in the short "fluid" phase, even if its length is much shorter than that of the "solid" phase.

In Fig. 3 we have plotted the final frequency reached after 1 min, i.e. at the end of the "fluid" phase, as a function of $\beta$, for different values of the magnetic field $B$. We see that while for low magnetic fields $\left(B \sim 10^{14} \mathrm{G}\right)$ the rotation frequency remains substantially unchanged, for larger fields the spin-down can be extremely fast and can slow down the magnetar to a frequency much smaller than the initial one. Moreover, we note that for a fixed $B$, the final frequency is not very strongly dependent on $\beta$. This is due to the fact that electromagnetic spin-down is always the dominant contribution to $\dot{\Omega}$ (and $\dot{\Omega}_{\mathrm{em}} \propto B^{2}$ ), except in the case of large values of $B$ and $\beta$ and when the initial few seconds of the star evolution are considered, see Fig. 2.

\section{Emission of gravitational waves}

In this section, we discuss the emission of gravitational radiation by a newborn magnetar, estimating its detectability by future interferometric detectors.

The gravitational signal from a distorted, fluid, rotating star is $h_{i j} \propto \ddot{\mathcal{I}}_{i j}$; from Eq. (6) we immediately see

\footnotetext{
4 This variation of $\epsilon$ introduces a new temporal dependency in $\dot{\Omega}_{\mathrm{gw}}$, Eq. (7), but when performing data analysis we can take this into account by re-defining the spin-down parameters.
} 


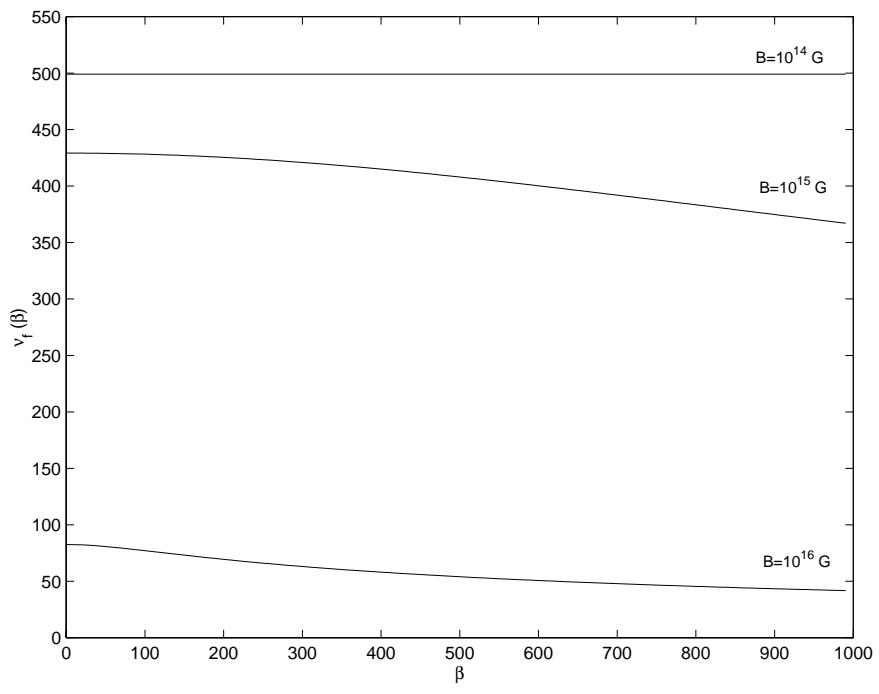

Fig. 3. Final rotation frequency reached 1 min after the birth of the magnetar as a function of the distortion parameter $\beta$. Different curves correspond to different values of the polar magnetic field $B$

that radiation will be emitted at two frequencies: $\nu$ and $2 \nu$. This is true also once the crust has solidified, then the following equations hold for both cases. Note that the angular velocity axis is coincident with the total angular momentum direction, while in a precessing star they are misaligned. From the relation $\frac{\mathrm{d} E}{\mathrm{~d} \nu}=\frac{\mathrm{d} E}{\mathrm{~d} t}\left(\frac{\mathrm{d} \nu}{\mathrm{d} t}\right)^{-1}$ and from Eqs. (6-8) we find that the power spectrum at the two frequencies is

$\left(\frac{\mathrm{d} E}{\mathrm{~d} \nu}\right)_{\nu}=\frac{K_{1} \cos ^{2} \alpha \nu^{3}}{K_{2}+K_{3} \nu^{2}}$

$\left(\frac{\mathrm{d} E}{\mathrm{~d} \nu}\right)_{2 \nu}=\frac{1}{8} \frac{K_{1} \sin ^{2} \alpha(2 \nu)^{3}}{K_{2}+\frac{K_{3}}{16}(2 \nu)^{2}}$

with

$K_{1}=\frac{16 \pi^{4}}{5} G \epsilon^{2} I_{z}^{2}$

$K_{2}=\frac{R^{6}}{3 I_{z}} B^{2}$

$K_{3}=4 \pi^{2} \frac{G}{c^{2}} \epsilon^{2} I_{z}\left(\frac{1}{5}+3 \sin ^{2} \alpha\right)$.

The two components of the gravitational signal are given by (Bonazzola \& Gourgoulhon 1996)

$$
\begin{aligned}
h_{+}(t) & =h_{0} \sin \alpha \\
& \times\left(\frac{1}{4} \cos \alpha \sin 2 \theta \cos \Omega t-\sin \alpha \cos ^{2} \frac{\theta}{2} \cos 2 \Omega t\right)
\end{aligned}
$$

and

$$
\begin{aligned}
h_{\times}(t)= & h_{0} \sin \alpha \\
& \times\left(\frac{1}{2} \cos \alpha \sin \theta \sin \Omega t-\sin \alpha \cos \theta \sin 2 \Omega t\right),
\end{aligned}
$$

where $\theta$ is the angle between the star rotation axis and the direction of observation.

Now we estimate the detectability of the gravitational waves emitted, calculating the corresponding signal-tonoise-ratio $(S N R)$. We use the formula for the $S N R$ in the case of optimum filtering, which presumes that we know exactly the waveform and that we have at our disposal, at least for some extreme values of the parameters, a huge computational power; thus our results must be actually considered as upper limits. The basic formula, after performing an average over the relative source-detector position and on the polarization of the waves, is

$\overline{S N R^{2}}=\frac{8 G}{5 \pi c^{3}} \int_{0}^{+\infty} \frac{f(\nu)}{\nu^{2} S_{\mathrm{h}}(\nu)} \mathrm{d} \nu$,

where $f(\nu)=\frac{1}{4 \pi d^{2}} \frac{\mathrm{d} E}{\mathrm{~d} \nu}$ is the one-sided energy spectrum, i.e. the flux of energy emitted per unit frequency ( $d$ is the distance between the source and the detector), and $S_{\mathrm{h}}(\nu)$ is the one-sided noise spectral density of the detector (units $1 / \mathrm{Hz}$ ).

We calculate separately the $S N R$ relative to the two phases of evolution of newborn magnetars, the "fluid" and the "solid" ones. For each of these, we calculate the $S N R$ for each frequency component of the signal, $S N R_{\nu}$ and $S N R_{2 \nu}$, and then combine them through a weighted average. The choice of the weights is outside the scope of the present paper and we will simply use $S N R=\sqrt{\frac{{\overline{S N R^{2}}}_{\nu}+{\overline{S N R^{2}}}_{2 \nu}}{2}}$.

Results of the $S N R$, as a function of $\beta$, are plotted in Figs. 4-6, respectively for the initial Virgo detector, for $L I G O-I I$ and $L I G O-I I I$. They have been calculated assuming a neutron star with mass $1.4 M_{\odot}$ and radius $R=12.5 \mathrm{~km}$ at a distance $d=20 \mathrm{Mpc}$. According to Kouveliotou et al. (1994), the formation rate of magnetars should be $\sim 10 \%$ that of normal neutron stars. This means that we expect $\sim 1 \mathrm{eV} / \mathrm{yr}$ within the distance of the Virgo cluster. For Virgo the following fit to the noise spectral density has been used (Canitrot et al., preprint):

$$
\begin{aligned}
S_{\mathrm{h}}(\nu)= & \frac{310^{-43}}{\nu}+\frac{510^{-37}}{\nu^{5}} \\
& +610^{-46}\left[1+\left(\frac{\nu}{500 \mathrm{~Hz}}\right)^{2}\right],
\end{aligned}
$$

while for the $L I G O$ detectors we have used the fits given in Owen et al. (1998).

For a fixed $B$, the relative values of $S N R$ for the "fluid" and the "solid" phase depend mainly on the frequency bands which they span. For example, for $B=10^{16} \mathrm{G}$, most of the frequency range $[10,500] \mathrm{Hz}$ is covered during the initial "fluid" phase and consequently most of the signal-to-noise ratio is accumulated here. However, for a magnetic field $B \simeq 10^{15} \mathrm{G}$, the final frequency reached at the end of the "fluid" phase is still rather large (see Fig. 3) and therefore the following "solid" phase weights more. We see that detection by the inital Virgo interferometer could be possible only for very large values of $\beta$ and strong fields. Detection perspectives are better for enhanced and 


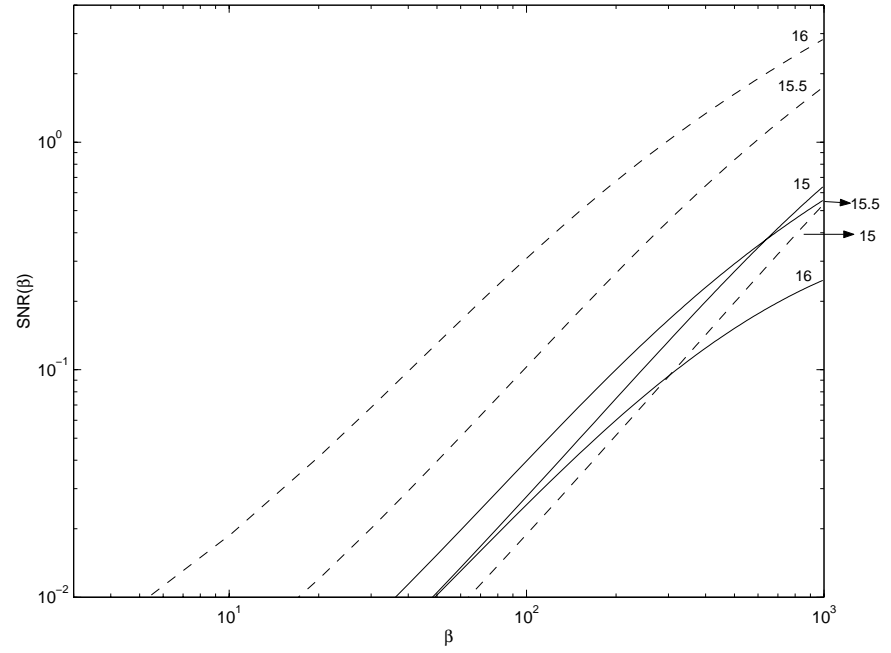

Fig. 4. $S N R$ as a function of the distortion parameter $\beta$ for the Virgo detector. The source is assumed to be at a distance $d=20$ Mpc. Dashed lines refers to the initial "fluid" phase of the magnetar evolution, while solid lines are relative to the following "solid" phase. Different curves correspond to different values of $\log (B)$

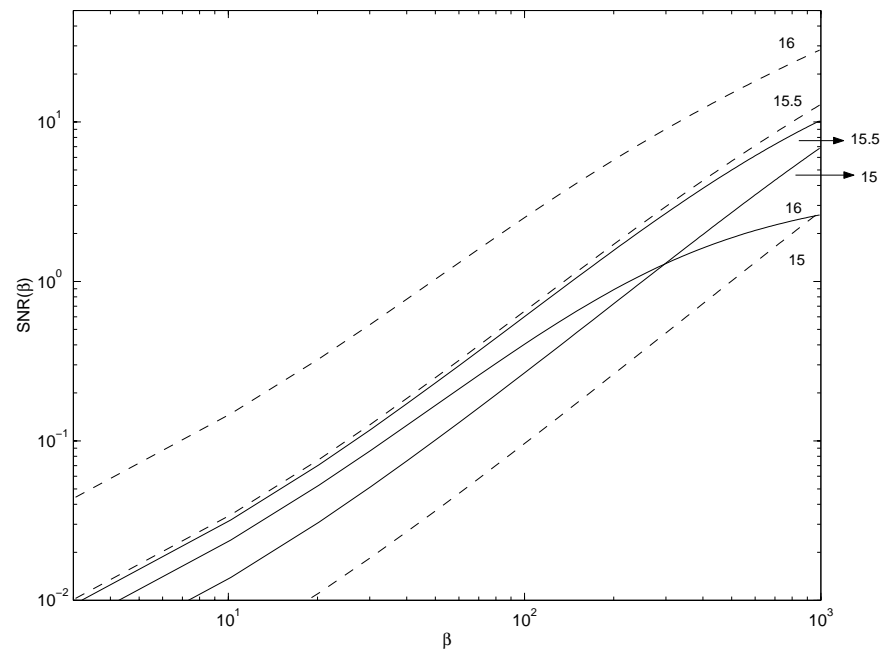

Fig. 5. The same as in Fig. 4, but relative to the $L I G O-I I$ detector

advanced detectors: for instance, for a $L I G O-I I$ detector we need $\beta>45(\beta>200)$ and $B \sim 10^{16} \mathrm{G}\left(B \sim 10^{15} \mathrm{G}\right)$ in order to have $S N R>1$. These values are reduced somewhat for a $L I G O-I I I$ detector. Clearly, an even larger $S N R$ could be obtained if the transition from the "fluid" to the "solid" phase were smooth and could be suitably modeled so that a single long stretch of data, covering both phases, could be used.

\section{Conclusions}

In this paper, we calculate the dynamical evolution of newborn magnetars, under the action of electromagnetic and gravitational torques, and estimate the detectability of the

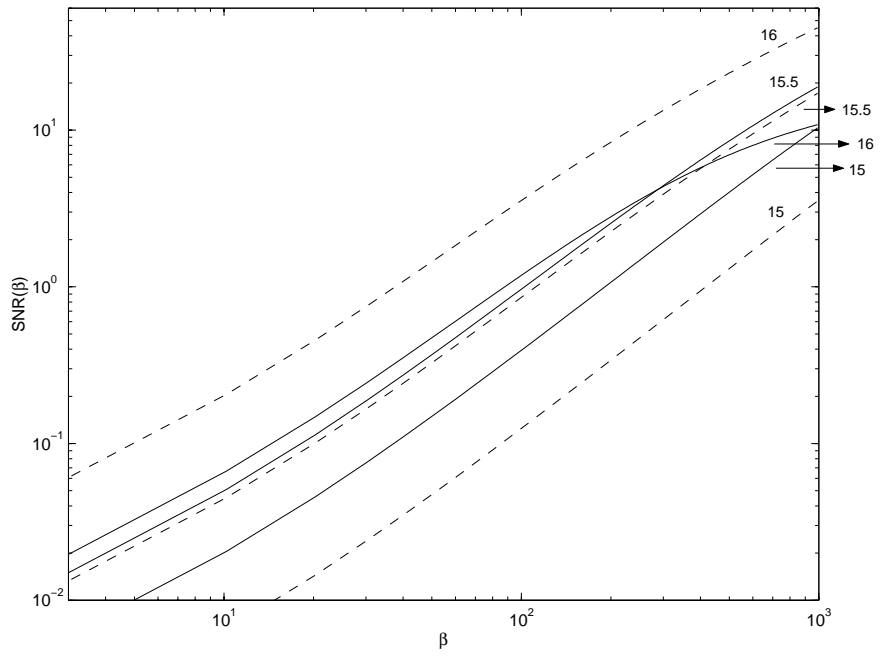

Fig. 6. The same as in Fig. 4, but relative to the $L I G O-I I I$ detector

gravitational waves they emit. We consider separately the inital "fluid" phase, which lasts $\sim 1 \mathrm{~min}$, and the following "solid" phase, which starts when the solid crust forms, until the rotation frequency is outside the sensitivity band of interferometers. We parametrize our results as a function of the magnetic field $B$ and of the distortion parameter $\beta$, which depends on the star equation of state and on the structure of the magnetic field inside the star. We calculate the signal-to-noise ratio using the sensitivity curves of the inital Virgo interferometer and of enhanced and advanced $L I G O$ detectors $(L I G O-I I, L I G O-I I I)$. We find that signals emitted by newborn magnetars in the Virgo cluster could be detected by Virgo for large values of $\beta(>300)$ and $B \sim 10^{16} \mathrm{G}$ and by advanced interferometers for moderate $\beta(>150)$ and $B>10^{15} \mathrm{G}\left(\beta>40\right.$ if $\left.B \sim 10^{16} \mathrm{G}\right)$. Such values of $\beta$ could occur in neutron stars, at least in principle. Our calculations are based on some simplifying assumptions, which reflect our lack of knowledge regarding the initial evolutionary phases of neutron stars with a strong magnetic field. However, our results are rather promising and suggest that a deeper study of the problem should be undertaken.

Among the main points which have to be taken into account, we note the stability conditions for a strongly magnetized and fast rotating neutron star (connected, for example, to the occurrence of secular or dynamical instabilities), the range of values for $\beta$ occurring in real neutron stars, the transition from the "fluid" to the "solid" phase and the behaviour of the solid crust when a strong magnetic field is present. Not less important is the problem of outlining a proper strategy for data analysis.

Acknowledgements. I would like to thank Sergio Frasca and Nikolaos Stergioulas for the useful suggestions and the anonymous referee for his/her comments. 


\section{References}

Bocquet, M., Bonazzola, S., Gourgoulhon, E., \& Novak, J. 1995, A\&A, 301, 757

Bonazzola, S., \& Gourgoulhon, E. 1996, A\&A, 312, 675

Chandrasekhar, S. 1969, Ellipsoidal Figures of Equilibrium (Yale University Press, New Haven)

Colpi, M., Geppert, U., \& Page, D. 2000, accepted for publication in ApJ

Duncan, R. C., \& Thompson, C. 1992, ApJ, 392, L9

Duncan, R. C., \& Thompson, C. 1995, AIP Conference, High velocity neutron stars and gamma-ray bursts, ed. R. E. Rothschild, \& R. E. Lingenfelter (AIP press)

Duncan, R. C. 1999, talk given at "Fifth Huntsville GammaRay Burst Symposium"

Goldreich, P., \& Reisenegger, A. 1992, ApJ, 395, 250
Goussard, J. O., Haensel, P., \& Zdunik, J. L. 1998, A\&A, 330, 1005

Konno, K., Obata, T., \& Kojima, Y. 2000, A\&A, 356, 234

Kouveliotou, C., Fishman, J. C., Meegan, C. A., et al. 1994, Lett. Nat.

Mereghetti, S. 1999, invited review at the NATO Advanced Study Institute "The Neutron Star-Black Hole Connection"

Owen, B. J., Lindblom, L., Cutler, C., Schutz, B. F., Vecchio, A., \& Andersson, N. 1998, Phys. Rev. D, 58, 084020

Palomba, C. 2000, A\&A, 354, 163

Ruderman, M. 1991, ApJ, 382, 576

Ruderman, M. 1991, ApJ, 382, 587

Thompson, C., \& Duncan, R. C. 1996, ApJ, 473, 322

Thompson, C., Duncan, R. C., Woods, P. M., et al. 1999, preprint [astro-ph/9908086] 